\title{
Spectral and Total Radiation Properties of Turbulent Carbon Monoxide/Air Diffusion Flames
}

\author{
J. P. Gore, ${ }^{*}$ S.-M. Jeng, $\dagger$ and G. M. Faeth $\ddagger$ \\ University of Michigan, Ann Arbor, Michigan
}

\begin{abstract}
A study of the structure and radiation properties of round turbulent carbon monoxide/air diffusion flames is described. Measurements of mean and fluctuating streamwise velocity and mean temperatures, concentrations of major gas species, spectral radiation intensities, and radiant heat fluxes were made. The measurements were used to evaluate predictions based on the laminar flamelet concept and narrow-band radiation models, both ignoring (using mean properties) and considering (using a stochastic method) the effects of turbulence/radiation interactions. State relationships were found by correlating auxiliary measurements in laminar flames. Structure and radiation predictions were reasonably good for present test conditions. The effects of turbulence/radiation interactions were small for these reactants (increasing spectal intensities ca. $10 \%$ ) since radiation properties vary slowly with mixture fraction near stoichiometric conditions.
\end{abstract}

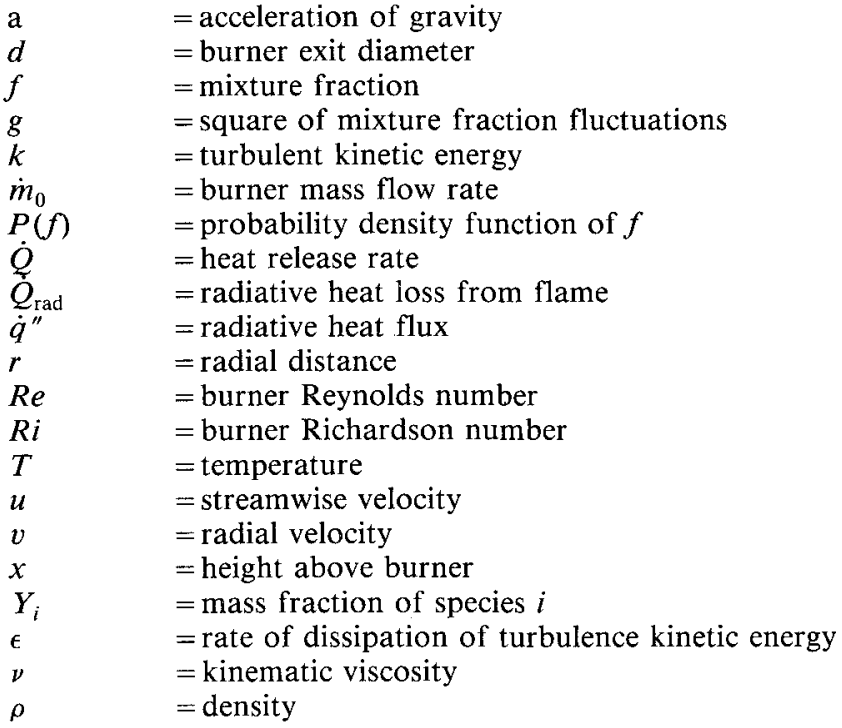

Subscripts

$$
\begin{aligned}
& c \quad=\text { centerline quantity } \\
& 0 \quad=\text { burner exit condition } \\
& \infty \quad=\text { ambient condition }
\end{aligned}
$$

Superscripts

$\left({ }^{-}\right),\left({ }^{-}\right)^{\prime}=$ time-averaged mean and fluctuating quantity

$(\sim),(\sim) "=$ Favre-averaged mean and fluctuating quantity

\section{Introduction}

T HIS study is an extension of earlier work on the structure and radiation properties of turbulent methane/air and propane/air diffusion flames. ${ }^{1-5}$ The present investigation

Presented as Paper 86-0294 at the AIAA 24th Aerospace Sciences Meeting, Reno, NV, Jan. 6-9, 1986; received Feb. 21, 1986; revision received June 17, 1986. Copyright (C) American Institute of Aeronautics and Astronautics, Inc., 1986. All rights reserved.

*Predoctoral Scholar, Department of Aerospace Engineering.

$\dagger$ Postdoctoral Fellow, Department of Aerospace Engineering; presently with Center for Laser Applications, University of Tennessee Space Institute, Tullahoma.

†Professor, Department of Aerospace Engineering. Associate Fellow AIAA. considers turbulent carbon monoxide/air diffusion flames both theoretically and experimentally. These reactants yield a nonluminous flame having relatively simple chemistry and radiation properties, which simplifies interpretation of the results. The objective of the study was to complete new measurements of the structure and radiation properties of turbulent carbon monoxide/air diffusion flames. Analysis was used to help interpret the measurements and to initiate the evaluation of models of the process. An aspect of particular interest was the effect of turbulent fluctuations on flame radiation properties, i.e., o examine potential errors when radiation predictions are based on mean properties, which is common practice. The following discussion is brief; additional details and a complete tabulation of the data are provided in Refs. 6 and 7.

We recently reviewed past work on the radiative properties of turbulent flames and this discussion will not be repeated. ${ }^{8}$ It was concluded that additional data were needed to support development of the analysis-particularly, results for welldefined experimental conditions having information on both structure and radiation properties. Another important issue for turbulent flames is the effect of turbulent fluctuations on flame radiation. Current practice generally ignores this interaction and mean scalar properties are used to predict radiation intensities and heat fluxes. ${ }^{8,9}$ Yet $\operatorname{Cox}^{10}$ and Kabashnikov and Kmit, ${ }^{11}$ using simplified analysis, found that turbulent fluctuations appreciably increase (up to 2-3:1) radiative heat fluxes in flames, in comparison to values based on mean properties.

The present study is an extension of work by Jeng and coworkers, ${ }^{1-5}$ who examined these same issues for methane/ air and propane/air diffusion flames. Measurements of flame structure and radiation properties were used to evaluate models of these phenomena. Structure was analyzed using a Favre-averaged $k-\epsilon-g$ turbulence model, which incorporates the conserved-scalar formalism, similar to the approach described by Bilger. ${ }^{12} \mathrm{~A}$ central element of this model is the use of the laminar flamelet approximation to relate scalar properties to mixture fraction. This approximation is based on observations by Bilger ${ }^{13}$ and Liew et al. ${ }^{14}$ that scalar properties in laminar diffusion flames can be correlated as universal functions of the mixture fraction for a wide range of flame stretch rates, showing departure from universality only near the extinction limits. Use of these ideals yielded reasonably good predictions of the scalar structure of turbulent methane and propane/air diffusion flames. ${ }^{3,4}$ 
Structure predictions were subsequently used to evaluate methods for predicting the spectral radiation intensities and radiant heat fluxes of turbulent methane/air diffusion flames. ${ }^{2,5}$ Spectral radiation intensities were predicted using a narrow-band model described by Ludwig et al., ${ }^{9}$ but with extensions to include the gas bands due to methane and carbon monoxide. Computations were made using both the mean properties along a path and a stochastic method to allow for turbulence radiation interactions. The stochastic method employed random-sampling techniques adapted from Shuen et al. ${ }^{15}$ The comparison between predictions and measurements was encouraging. The differences between the mean property and stochastic predictions suggested only modest effects of turbulence/radiation interactions, e.g., stochastic predictions were generally only $20-30 \%$ higher than mean property predictions. Such differences are on the order of uncertainties in the narrow-band and stucture models. Summing the spectral radiation intensity predictions over both wavelengths and the radiation paths covering all portions of the flame seen by the detector, using the discretetransfer method described by Lockwood and Shah, ${ }^{16}$ also yielded reasonably good predictions of radiative heat fluxes.

The objective of the present investigation was to test these ideas further by completing new measurements for turbulent carbon monoxide/air diffusion flames. Mean and fluctuating velocities, mean temperatures, concentrations of major gas species, spectral radiation intensities, and radiative heat fluxes were measured for two turbulent carbon monoxide/air diffusion flames. These results, along with recent structure measurements by Razdan and Stevens ${ }^{17}$ for the same reactants, were used to evaluate predictions. Structure measurements in laminar carbon monoxide/air diffusion flames were also completed to develop state relationships needed by the analysis.

The paper begins with discussion of experimental and theoretical methods. Results concerning the state relationship measurements and the structure of the turbulent diffusion flames are then described. The paper concludes with descriptions of predicted and measured spectral radiation intensities and radiative heat fluxes.

\section{Experimental Methods}

\section{Test Arrangement}

Experimental methods were generally similar to past work and will be discussed here only briefly. ${ }^{1-5}$ The turbulent diffusion flames involved vertical injection of carbon monoxide from a water-cooled burner into still air. The burner had a screened plenum followed by a $25: 1$ contraction, which terminated in a 5 -mm-diam exit passage. The flames were attached at the burner exit using a small coflow of hydrogen from a slot just below the exit in the burner passage. The burner was housed in a screened enclosure to reduce room disturbances. The burner could be traversed in three directions to accommodate rigidly mounted optical instrumentation.

An uncooled concentric-tube burner was used for the laminar flame measurements. The carbon monoxide flowed from a central tube $(14.3 \mathrm{~mm}$ in diameter) with a coflow of air from a concentric port having an inside diameter of 102 $\mathrm{mm}$. The burner flows were smoothed using a bed of stainless steel balls and honeycomb ( $1 \mathrm{~mm}$ hexagonal cells by $25 \mathrm{~mm}$ long) that was flush with the burner exit. The flames were shielded from drafts with a concentric cylindrical quartz tube (115 $\mathrm{mm}$ i.d.) that extended $10 \mathrm{~mm}$ beyond each measuring plane. Fine-mesh screen was used to shield the upper portions of the flame.

\section{Instrumentation}

Velocities

Mean and fluctuating velocities were measured using a single-channel laser Doppler anemometer (LDA). The LDA used a $50-\mathrm{mW}$ HeNe laser and was operated in the dualbeam forward-scatter mode. Frequency shifting was used to eliminate directional bias and ambiguity. The seeding levels were high; therefore, the analog output of the burst counter signal processor was integrated directly to yield time averages without velocity bias. The measuring volume had a diameter of $0.24 \mathrm{~mm}$ and a length of $0.72 \mathrm{~mm}$. Analysis indicated that gradient broadening effects were small, except near the downstream end of the potential core. Uncertainties $(95 \%$ confidence) of mean and fluctuating velocities were less than $5 \% .^{7}$

\section{Temperatures}

The mean temperatures were measured using a buttwelded thermocouple constructed out of 0.075 -mm-diam $\mathrm{Pt} / \mathrm{Pt}-10 \% \mathrm{Rh}$ wires. The region of the junction was somewhat enlarged, having a diameter of roughly $0.2 \mathrm{~mm}$. The junction wires were mounted on heavier lead wires, spaced $12 \mathrm{~mm}$ apart to provide a traversable probe. The thermocouple output was recorded with an integrating digital voltmeter. Temperature measurements were not corrected for radiative heat losses and are estimated to be $100-200 \mathrm{~K}$ too low in the hottest parts of the flames.?

\section{Species Concentrations}

Mean gas concentrations were measured in the turbulent flames using isokinetic sampling (at the mean gas velocity) and analysis with a gas chromatograph. A water-cooled probe with an inlet diameter of $2 \mathrm{~mm}$ was used. Samples were analyzed using a gas chromatograph having a hot-wire detector, which was calibrated with known gas mixtures; see Ref. 7 for full details concerning these measurements. Aside from uncertainties in the degree of density weighting for sampling in turbulent flames, to be quantified later, uncertainties in composition measurements are less than $15 \%{ }^{7}$

Concentration measurements in the laminar flames employed a quartz sampling probe, operated choked, having a

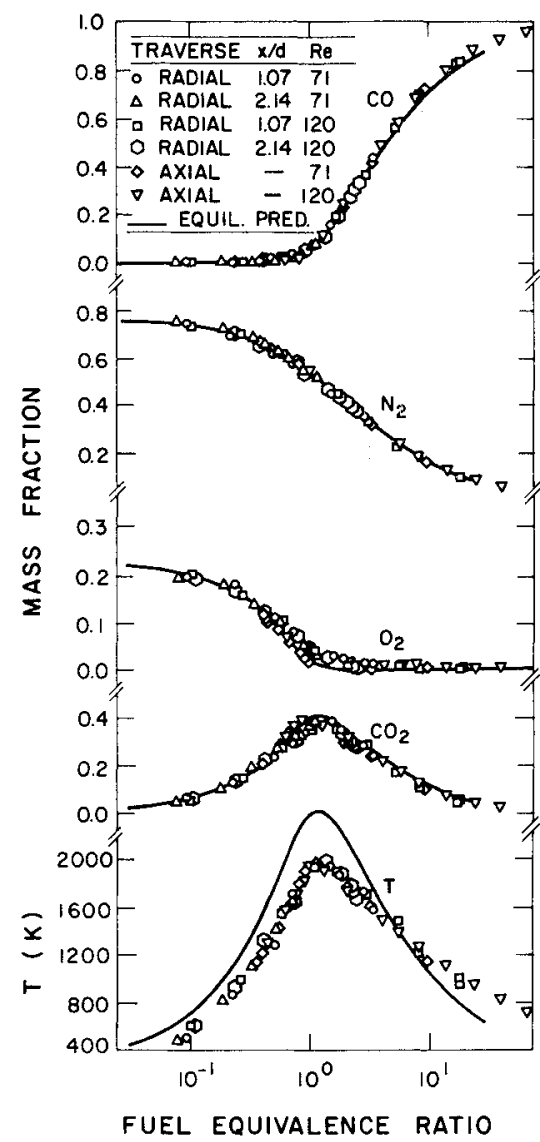

Fig. 1 Carbon monoxide/air state relationships. 
Table 1 Summary of turbulent flame test conditions ${ }^{a}$

\begin{tabular}{|c|c|c|c|}
\hline & \multicolumn{3}{|c|}{ Reynolds no. } \\
\hline & 13,140 & 7475 & 11,400 \\
\hline Source & Present study & Present study & Ref. 17 \\
\hline $\begin{array}{l}\text { Richardson no. } \\
\text { Measured }\end{array}$ & $2.3 \times 10^{-5}$ & $7.9 \times 10^{-5}$ & $3.9 \times 10^{-5}$ \\
\hline$u_{0} \mathrm{~m} / \mathrm{s}$ & 51.5 & 24.8 & 35.5 \\
\hline$k_{0}^{1 / 2} / u_{0}$ & 0.051 & 0.058 & - \\
\hline$Q, \mathrm{~kW}$ & 8.9 & 5.3 & 7.6 \\
\hline $\bar{Q}_{\mathrm{rad}} / Q, \%$ & 8.4 & 11.4 & - \\
\hline \multicolumn{4}{|l|}{ Flow rates, $\mathrm{mg} / \mathrm{s}$} \\
\hline Fuel & 895 & 531 & 760 \\
\hline Hydrogen & 19.2 & 4.8 & 1.7 \\
\hline
\end{tabular}

${ }^{a}$ Flow directed vertically upward from a 5 -mm-diam passage, in still air a NTP (except for the Razdan and Steven ${ }^{17}$ flame where there was a $0.13 \mathrm{~m} / \mathrm{s}$ coflow of air). Commerical grade carbon monoxide, Linde Division of Union Carbide, containing $1.12 \%$ hydrogen by volume. ${ }^{\mathrm{b}} \mathrm{Re}=u_{0} d / \nu$ based on fuelgas properties at exit. ${ }^{c} R i=a d / u_{0}^{2}$.

sampling orifice of $0.1-0.2 \mathrm{~mm}$ diam and a rounded probe tip with a radius of $0.75 \mathrm{~mm}$. The aspect ratio of these measurements was ca. 50-100, concentration profiles had low levels of curvature, and results are considered in terms of mixture fraction rather than position; therefore, effects of gradient broadening are small in comparison to other uncertainties. Chromatography methods and uncertainties were similar to the turbulent flame measurements.

\section{Spectral Radiation Intensities}

Spectral radiation intensities were measured for radial paths through the flames using a $250-\mathrm{mm}$ grating monochromator with a pyroelectric detector (Oriel Corp., models 7240 and 7084). The field of view was roughly $10 \mathrm{~mm}$ in diameter with a $1.2 \mathrm{deg}$ field angle. A water-cooled aperture between the flame and the monochromator prevented overheating of the instrument. Various gratings and order-sorting filters were used to cover the wavelength range 2.4-6.0 $\mu \mathrm{m}$, which includes the 2.7 and $4.3-\mu \mathrm{m}$ bands of carbon dioxide and the 4.7- $\mu \mathrm{m}$ band of carbon monoxide. The system function of the monochromator was calibrated using a blackbody source heated by a furnace. Higher-order harmonics of known wavelengths from a mercury arc and a $\mathrm{HeNe}$ laser were used to calibrate the wavelengh readout. Uncertainties in these measurements were less than $20 \%$. $^{7}$

\section{Radiative Heat Fluxes}

Total radiative heat flux distributions were measured along the base and the axis of the turbulent flames using a gas-purged, water-cooled sensor (Medtherm, type 64P-10-22, with a $150 \mathrm{deg}$ viewing angle). The sensor was operated with a sapphire window, which has negligible transmittivity above $6 \mu \mathrm{m}$, eliminating gas bands outside the range of the spectral intensity calculations. The sensor was positioned so that the flame boundaries were entirely within its viewing angle, except for points far from the burner exit, which contribute very little to the radiative flux in any event. Uncertainties of these measurements were less than $10 \%{ }^{7}$

\section{Test Conditions}

Test conditions for the turbulent flames are summarized in Table 1. Both the present flames and the flame considered by Razdan and Steven ${ }^{17}$ are shown, since the latter flame was also used to evaluate structure predictions. Relatively high burner exit Reynolds numbers were used to assure turbulent flow. The initial Richardson numbers were relatively low; nevertheless, the effects of buoyancy were still appreciable, particularly near the end of the visible flame zone for the lowest Reynolds number flame.
Initial conditions for the present flames were defined by measuring $\bar{u}, \bar{u}^{\prime}$, and $\bar{v}^{\prime}$ at $x / d=2$, which was the position learest the exit accessible to the LDA. The initial turbulence kinetic energy distribution was estimated from these measurements assuming that the radial and tangential velocity fluctuations were equal. Initial values of $\epsilon$ were estimated from the rate of decay of velocity fluctuations in the potential core.

\section{Theoretical Methods}

\section{Flame Structure}

Theoretical methods were similar to past practice and will be described only briefly. ${ }^{2-5}$ The conserved-scalar method was used to estimate flame structure, assuming 1) boundarylayer approximations for an axisymmetric flame with no swirl, 2) negligible mean kinetic energy, 3) equal exchange coefficients for all species and heat, 4) negligible radiative energy exchange within the flames, and 5) buoyancy only affects the mean flow. Assumptions 1 and 2 represent conditions of the experiments. Assumption 3 is widely adopted for analysis of turbulent mixing in gases and is reasonably satisfactory at high Reynolds numbers. Assumption 4 is justified by the relatively small radiative heat loss fractions of present flames (see Table 1). Assumption 5 has been shown to provide reasonably good predictions of mean properties in turbulent diffusion flames having similar initial conditions. ${ }^{3,4}$ The Favre-averaged formulation of Bilger ${ }^{12}$ was adopted (although details differ), which involves solving governing equations for mean conservation of mass, momentum, and mixture fraction and modeled governing equations for $k, \epsilon$, and $g$. All model constants were fixed by measurements in constant- and variable-density noncombusting jets. ${ }^{3}$

Scalar properties were found from the probability density function of mixture fraction in conjunction with the laminar flamelet state relationships that provide scalar properties as a function of mixture fraction. A clipped Gaussian Favre probability density function was used, similar to past work. ${ }^{3,4}$ The laminar flamelet state relationships were only used for the mass fractions of major species. Temperatures and densities were computed as a function of mixture fraction, for the measured compositions, assuming that the flame lost the measured fraction of its chemical energy release by radiation. The calculations for temperature and density used the Gordon and $\mathrm{McBride}^{19}$ chemical equilibrium.

\section{Spectral Radiation Intensities}

The equation of radiative transfer was solved for various paths through the flame, ignoring scattering using the narrow-band analysis of Ludwig et al. ${ }^{9}$ The analysis considered all gas bands in the region of interest, e.g., the 2.7 . and $4.3-\mu \mathrm{m}$ bands of carbon dioxide and the $4.7-\mu \mathrm{m}$ band of carbon monoxide, using the Goody statistical narrow-band model in conjunction with the Curtis-Godson approximation for an inhomogeneous gas path. A slightly modified version of the computer algorithm RADCAL, developed by Grosshandler, ${ }^{18}$ was used for the computations.

Spectral intensity computations are straightforward when turbulence/radiation interactions are ignored. The structure analysis provides both time- and Favre-averaged mean scalar properties along the radiation path. Properties needed for the calculations are taken to be time averages.

The stochastic analysis was used to gain insight concerning turbulence/radiation interactions. This involves dividing the gas path into eddies, having lengths equal to the local dissipation length scale. Each eddy is assumed to have uniform properties. The properties of each eddy are found by randomly sampling its time-averaged probability density function for mixture fraction and obtaining scalar properties for this mixture fraction from the state relationships. Once all eddy properties are specified along the gas path, the spec- 
tral intensities are calculated similar to the mean property method. Random sampling continues in this manner until sufficient realizations have been computed to obtain statistically significant results.

\section{Radiative Heat Fluxes}

Computations of radiative heat fluxes are a straightforward extension of the spectral radiation intensity computations when the discrete transfer method of Lockwood and Shah ${ }^{16}$ is used. This involves determining the spectral intensity for various wavelengths and paths passing through the point in question (120 paths to the sensor location in this instance, allowing for symmetry) and then summing over both wavelengths and paths to find the total radiative heat flux. The paths are chosen to cover the region viewed by the detector with the contribution of each weighted according to the solid angle it intercepts and the angle of the path normal to the detector surface. The present calculations were based on mean property predictions to shorten the calculations, since turbulence/radiation interactions were not found to be large for carbon monoxide/air diffusion flames.

\section{Results and Discussion}

\section{State Relationships}

Measurements of species concentrations and temperatures in the laminar carbon monoxide/air diffusion flames are illustrated in Fig. 1. The measurements are plotted as a function of fuel equivalence ratio; however, mixture fraction is a single-valued function of this variable. The fuel equivalence ratio is found from the measured carbon and oxygen concentrations in the sample. Measurements are identified by the type of traverse from which they were obtained (radial at various heights or along the axis) and by the burner
Reynolds number (based on the fuel flow). Specific positions for each value can be obtained from the data tabulation.? Equilibrium predictions for an adiabatic flame, from the Gordon and $\mathrm{McBride}{ }^{19}$ code, are also plotted in the figures.

It is evident that the measurements correlate as a function of fuel equivalence ratio, supporting the laminar flamelet concept for these test conditions. Aside from temperature (where radiative and convective heat losses from the flame and radiation errors of the thermocouple are factors), the correlations are in excellent agreement with predictions of local thermodynamic equlibrium. Thus, equilibrium predictions could be used to find the state relationships for this fuel system. This behavior is not usually observed for carbon dioxide and carbon monoxide in the fuel-rich regions of hydrocarbon flames where the oxidation of carbon monoxide to carbon dioxide does not proceed very rapidly in the presence of fuel molecules. ${ }^{20}$ In the present case, the capability of the system to approach theormodynamic equilibrium provides a physical justification for the flamelet concept. Figure 1 represents the state relationships for the species concentrations used during subsequent structure and radiation computations. The state relationship for temperature was obtained from chemical equilibrium computations ${ }^{19}$ with $10 \%$ of the total energy lost by radiation.

\section{Flame Structure}

\section{Present Measurements}

Structure predictions and measurements along the axis of the present turbulent diffusion flames are illustrated in Figs. 2 and 3. Time-averaged streamwise mean and fluctuating velocities were measured, while the predictions are Favre averages. The difference between the two for the mean velocities in the flames is typically not large, in comparison to experimental uncertainties, ca. $10 \% .{ }^{21,22}$ However, Favre-
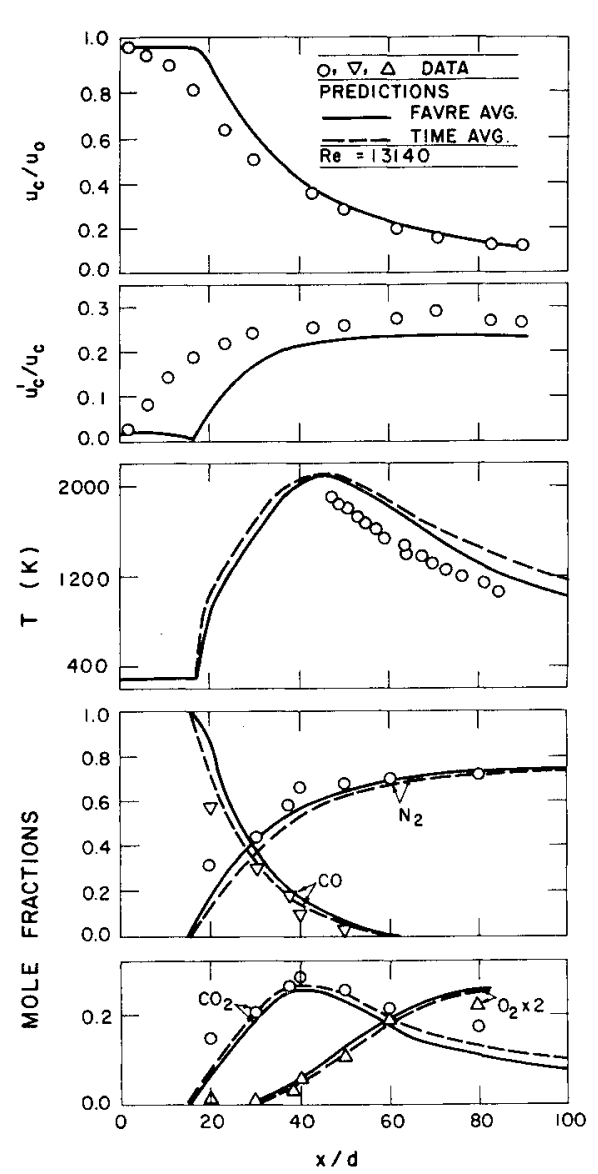

Fig. 2 Structure predictions and measurements along the axis, $\operatorname{Re}=13,140$.
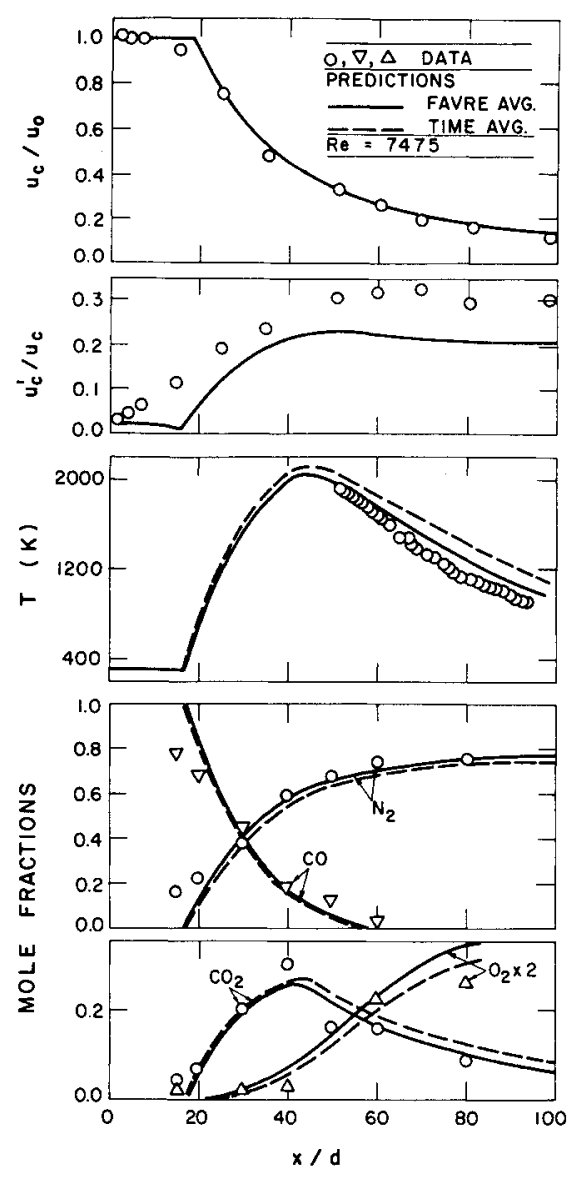

Fig. 3 Structure predictions and measurements along the axis, $\operatorname{Re}=\mathbf{7 4 7 5}$. 
averaged velocity fluctuations along the axis are on the order of $20 \%$ lower than time averages and can be as much as $40 \%$ lower in high-temperature portions of the flow. ${ }^{21,22}$ The analysis only yields $k$; therefore, $\bar{u}^{\prime \prime}$ has been estimated for plotting in Figs. 2 and 3 , assuming isotropic turbulence $\left(\tilde{u}^{\prime \prime 2}=2 k / 3\right)$, which is consistent with other model assumptions. If the usual level of anisotropy in jets is assumed $\left(\tilde{u}^{\prime \prime 2}=k\right)$, predictions would be roughly $20 \%$ higher. Mean velocities along the axis are predicted reasonably well for both flames, except near the end of the potential core where gradient biasing tends to reduce the measured mean velocities. Similar difficulties are encountered near the end of the potential core for velocity fluctuations. The predicted values of $\tilde{u}_{c}$ " are generally lower than the measured values of $\bar{u}_{c}^{\prime}$. However, this is consistent with expected lower values of the Favre averages and the usual levels of anisotropy of velocity fluctuations in jet-like flows, as noted earlier.

The analysis provides both time- and Favre-averaged scalar properties; therefore, both types of averages are illustrated in Figs. 2 and 3, since the degree of density weighting is uncertain for these measurements. For present conditions, however, differences between these averages are not very large. The comparison between predicted and measured concentrations is reasonably good. The temperature measurements were limited to positions downstream of the maximum temperature region due to problems with probe durability. In the region where measurements could be made, the comparison between the predicted and measured temperatures is reasonable, allowing for the fact that the measurements are not corrected for radiative heat losses and are probably $100-200 \mathrm{~K}$ too low in the highest temperature regions.

\section{Razdan and Stevens Measurements}

Predicted and measured mean temperatures and compositions for the flame considered by Razdan and Stevens ${ }^{17}$ are illustrated in Fig. 4. Only Favre-averaged concentration predictions are shown; however, differences between Favreand time-averaged concentrations are relatively small for

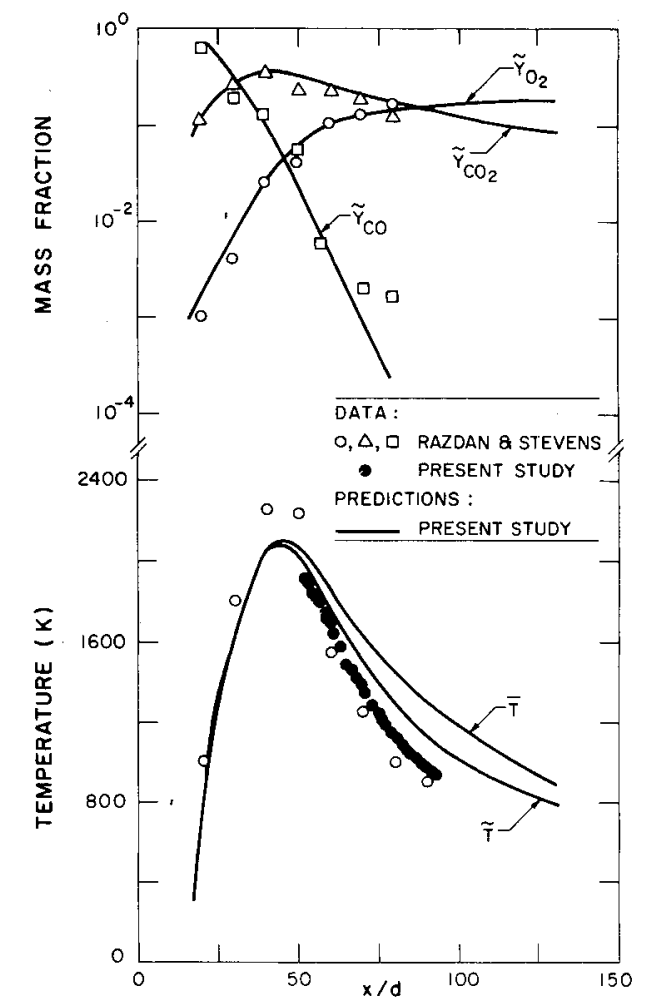

Fig. 4 Mean species mass fractions and temperatures along the axis, $R e=11,400$. these properties, similar to Figs. 2 and 3. The comparison between predictions and measurements is quite good.

Mean temperature predictions using both types of averaging are illustrated in Fig. 4. Predicted maximum temperatures underestimate the measurements, which were corrected for radiative heat losses. ${ }^{17}$ In view of the reasonably good predictions of other scalar properties, the reason for these discrepancies is unknown. However, the discrepancies are within typical uncertainties for measurements and predictions of this type in flames.

\section{Spectral Radiation Intensities}

The structure evaluation suggests that the present model provides reasonable predictions of mean properties in the present flames; therefore, we turn to consideration of radiation properties. Measurements and predictions of spectral radiation intensities for the present two flames are illustrated in Figs. 5 and 6. Measurements were undertaken for horizontal radial radiation paths through the flames at $x / d=50$ and 90 , the former position being very close to the flame tip. Both the mean property and stochastic predictions appear in the figures.

The results in Figs. 5 and 6 show that the spectral radiation intensities are dominated by the $2.7-$ and $4.3-\mu \mathrm{m}$ bands of carbon dioxide, although the $2.7-\mu \mathrm{m}$ band is not very apparent at $x / d=90$. Concentrations of carbon monoxide are small at the positions considered (see Figs. 2 and 3);

Fig. 5 Spectral radiation intensities, $R e=13,140$.

Fig. 6 Spectral radiation intensities, $\operatorname{Re}=\mathbf{7 4 7 5}$.
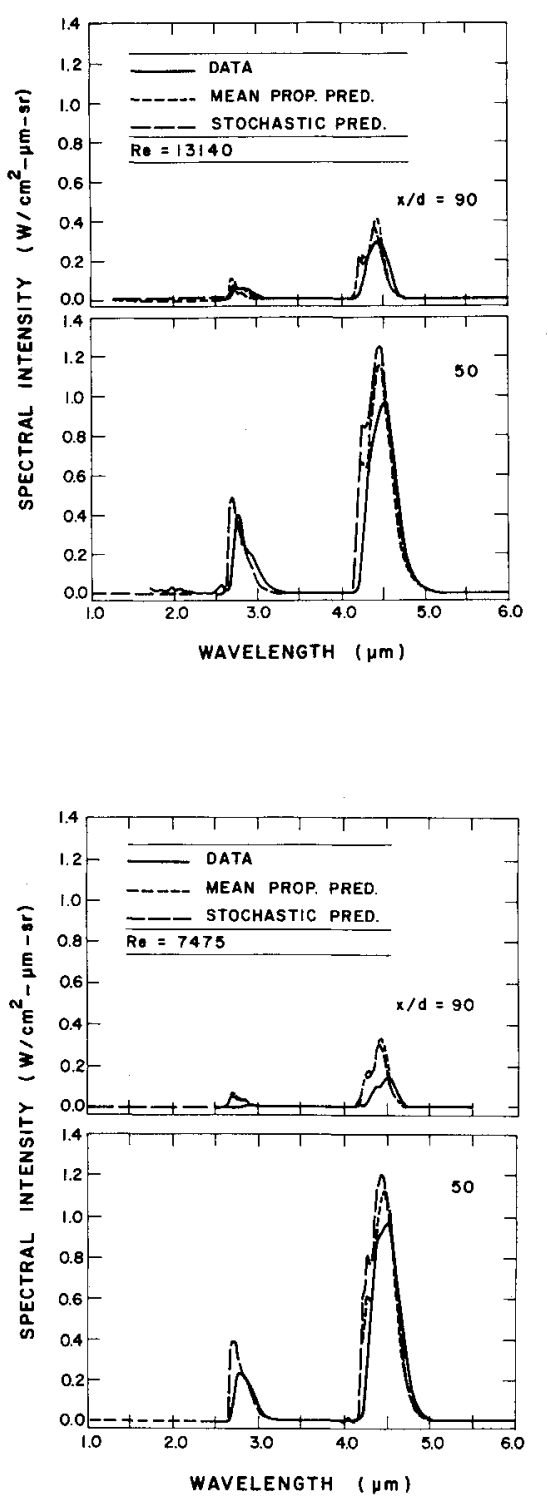


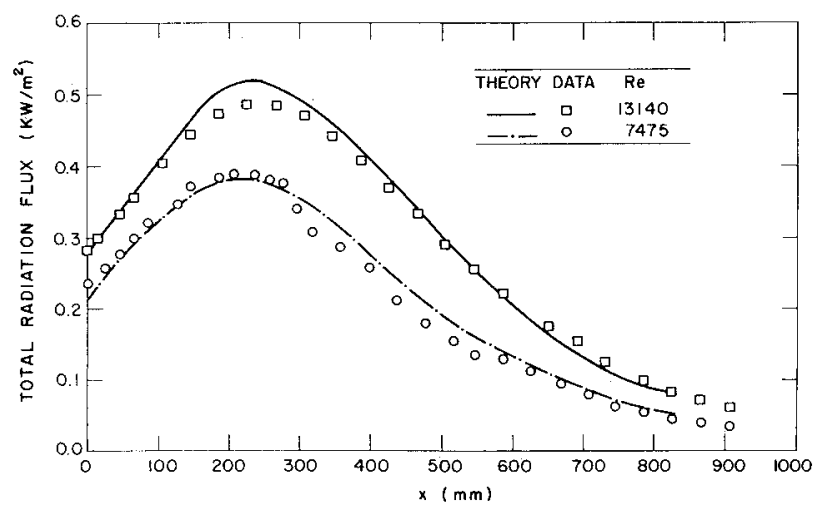

Fig. 7 Total radiative heat fluxes along axis.

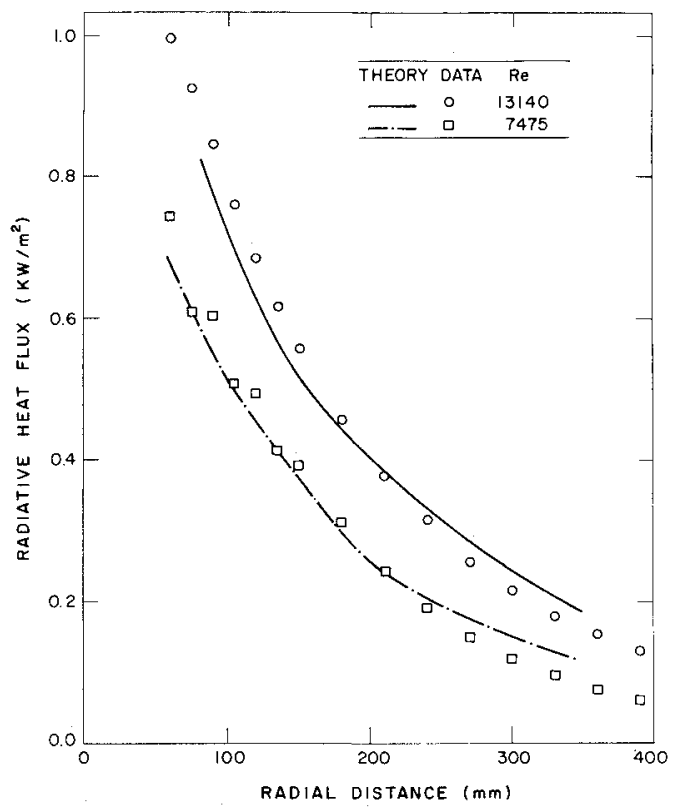

Fig. 8 Total radiative heat fluxes in the plane of the burner exit.

therefore, the $4.7-\mu \mathrm{m}$ band of carbon monoxide is not observed. Spectral radiation intensities are highest near the flame tip, e.g., $x / d=50$, where temperatures and concentrations of carbon dioxide are both high. The comparison between predictions and measurements is encouraging, particularly since the predictions depend on estimates of both the structure and radiation properties.

Effects of turbulence/radiation interactions are not large for the results illustrated in Figs. 5 and 6, e.g., stochastic predictions are only about $10 \%$ higher than the mean property predictions. In constrast, turbulence/radiation interactions caused the stochastic predictions to be $20-30 \%$ higher than the mean property predictions for turbulent methane/air diffusion flames. ${ }^{2}$ This is due to the relatively slow variation of radiation properties (viz., temperature and carbon dioxide concentration) with mixture fraction in the dominant region, near the stoichiometric condition, for this reactant combination. This behavior follows since the stoichiometric mixture fraction of carbon monoxide/air diffusion flames is substantially higher than hydrocarbon fuels (0.29 instead of 0.06$)$.

\section{Radiative Heat Fluxes}

Measurements and predictions of total radiative heat fluxes to points surrounding the present turbulent flames are illustrated in Figs. 7 and 8. Figure 7 is an illustration of results for the detector facing the flame axis and traversing in the vertical direction at a distance of $300 \mathrm{~mm}$ from the axis. The radiative heat flux is highest near the flame tip, ca. $x / d=45$ and 50 for the flow and high Reynolds number flames. Figure 8 is an illustration of results for a detector facing vertically upward in the plane of the burner exit and traversing radially outward. In this case, the radiative heat flux decreases monotonically with increasing radial distance. Although the peak mean temperatures and compositions are the same for both flames, the radiative heat fluxes are lower for the lower Reynolds number flame due to its smaller dimensions, e.g., buoyancy causes the flames to be shorter and narrower at lower Reynolds numbers for the present test range. This effect would disappear at higher Reynolds numbers, where the flame structure is relatively independent of Reynolds number. Predictions are in reasonably good agreement with measurements, e.g., discrepancies are on the order of $10 \%$.

\section{Conclusions}

The major conclusions of the study are as follows:

1) For the range of conditions examined here, state relationships of carbon monoxide/air diffusion flames are nearly universal and very close to predictions based on local thermodynamic equilibrium, supporting the laminar flamelet concept.

2) The present conserved-scalar $k-\epsilon-g$ analysis for structure was in reasonably good agreement with measurements, using measured initial conditions with all empirical constants fixed by results for noncombusting jets.

3) The present radiation analysis achieved reasonably good predictions of spectral radiation intensities and radiative heat fluxes, similar to past experience for turbulent methane/air diffusion flames., ${ }^{2,5}$ The effects of turbulence/radiation interactions are small for the carbon monoxide/air flames, increasing spectral radiation intensities on the order of only $10 \%$, due to the relatively slow variation of radiative properties with mixture fraction for this flame system.

\section{Acknowledgments}

This research was carried out while the authors were members of the Department of Mechanical Engineering at Pennsylvania State University, University Park. The research was supported by the U.S. Department of Commerce, National Bureau of Standards, Grant 60NANB4D0032, with B. J. McCaffrey of the Center for Fire Research serving as Scientific Officer.

\section{References}

${ }^{1}$ Jeng, S.-M., Chen, L.-D., and Faeth, G. M., "The Structure of Buoyant Methane and Propane Diffusion Flames," Nineteenth Symposium (International) on Combustion, The Combustion Institute, Pittsburgh, PA, 1982, pp., 349-358.

${ }^{2}$ Jeng, S.-M., Lai, M.-C., and Faeth, G. M., "Nonluminous Radiation in Turbulent Buoyant Axisymmetric Flames," Combustion Science and Technology, Vol. 40, Sept. 1984, pp. 41-53.

${ }^{3}$ Jeng, S.-M. and Faeth, G. M., "Species Concentrations and Turbulence Properties in Buoyant Methane Diffusion Flames," Journal of Heat Transfer, Vol. 106, Nov. 1984, pp. 721-727.

${ }^{4}$ Jeng, S.-M. and Faeth, G. M., "Predictions of Mean and Scalar Properties in Turbulent Propane Diffusion Flames," Journal of Heat Transfer, Vol. 106, Nov. 1984, pp. 891-893.

${ }^{5}$ Jeng, S.-M. and Faeth, G. M., "Radiative Heat Fluxes Near Turbulent Buoyant Methane Diffusion Flames," Journal of Heat Transfer, Vol. 106, Nov. 1984, pp. 886-888.

${ }^{6}$ Gore, J. P. and Faeth, G. M., "Radiation from Turbulent Flames," Final Report, NBS Grant 60NANB4D0032, Dept. of Mechanical Engineering, Pennsylvania State University, University Park, 1985. 
${ }^{7}$ Gore, J., "A Theoretical and Experimental Study of Turbulent Flame Radiation,"' Ph.D. Thesis, Pennsylvania State University, University Park, 1986.

${ }^{8}$ Faeth, G. M., Jeng, S.-M., and Gore, J. P., "Radiation from Flames," Heat Transfer in Fire and Combustion Systems, edited by C. K. Law, Y. Jaluria, W. W. Yuen, and K. Miyasaka, HTD Vol. 45, ASME, New York, 1985, pp. 137-151.

${ }^{9}$ Ludwig, C. B., Malkmus, W., Reardon, J. E., and Thomson, J. A., "Handbook of Infrared Radiation from Combustion Gases," NASA SP-3080, 1973.

${ }^{10}$ Cox, G., "On Radiation Heat Transfer from Turbulent Flames," Combustion Science Technology, Vol. 17, Oct. 1977, pp. 75-78.

${ }^{11}$ Kabashnikov, V. P. and Kmit, G. I., "Influence of Turbulent Fluctuations on Thermal Radiation," Journal of Applied Spectroscopy, Vol. 31, Aug. 1979, pp. 963-967.

${ }^{12}$ Bilger, R. W., "Turbulent Jet Diffusion Flames," Progress in Energy and Combustion Science, Vol. 1, Jan. 1976, pp. 87-109.

${ }^{13}$ Bilger, R. W., "Reaction Rates in Diffusion Flames," Combustion and Flame, Vol. 30, 1977, pp. 277-284.

${ }^{14}$ Liew, S. K., Bray, K. N. C., and Moss, J. B., "A Flamelet Model of Turbulent Non-Premixed Combustion," Combustion Science and Technology, Vol. 27, Dec. 1981, pp. 69-73.

${ }^{15}$ Shuen, J.-S., Solomon, A. S. P., Zhang, Q.-F., and Faeth, G. M., "Structure of Particle-Laden Jets: Measurements and Predictions," AIAA Journal, Vol. 23, March 1985, pp. 396-404.

\begin{abstract}
${ }^{16}$ Lockwood, F. C. and Shah, N. B., "A New Radiation Solution Method for Incorporation in General Combustion Prediction Procedures," Eighteenth Symposium (International) on Combustion, The Combustion Institute, Pittsburgh, PA, 1981, pp. 1405-1414.

${ }^{17}$ Razdan, M. K. and Stevens, J. G., "CO/Air Turbulent Diffusion Flame: Measurements and Modeling," Combustion and Flame, Vol. 59, March 1985, pp. 289-301.

${ }^{18}$ Grosshandler, W. L., "Radiative Heat Transfer in Nonhomogeneous Gases: A Simplified Approach," International Journal of Heat and Mass Transfer, Vol. 23, 1980, pp. 1447-1459.

${ }^{19}$ Gordon, S. and McBride, B. J., "Computer Program for Calculation of Complex Chemical Equilibrium Compositions, Rocket Performance, Incident and Reflected Shocks, and ChapmanJouget Detonations," NASA SP-273, 1971.

${ }^{20}$ Westbrook C. K. and Dryer, F. L., "Chemical Kinetic Modeling of Hydrocarbon Combustion," Progress in Energy and Combustion Science, Vol. 10, 1984, pp. 1-57.

${ }^{21}$ Faeth, G. M. and Samuelson, G. S., "Fast-Reaction Nonpremixed Combustion,"' Simple Turbulent Reacting Flows: Evaluation of Data for Model Predictions, edited by W. C. Strahle and S. G. Lekoudis, AFOSR TR-85 0880, Sept. 1985.

${ }^{22}$ Starner, S. H. and Bilger, R. W., "Measurements of ScalarVelocity Correlations in a Turbulent Diffusion Flame," Eighteenth Symposium (International) on Combustion, The Combustion Institute, Pittsburgh, PA, 1981, pp. 921-930.
\end{abstract}

\title{
From the AIAA Progress in Astronautics and Aeronautics Series
}

\section{RAREFIED GAS DYNAMICS—v. 74 (Parts I and II)}

\author{
Edited by Sam S. Fisher, University of Virginia
}

The field of rarefied gas dynamics encompasses a diverse variety of research that is unified through the fact that all such research relates to molecular-kinetic processes which occur in gases. Activities within this field include studies of (a) molecule-surface interactions, (b) molecule-molecule interactions (including relaxation processes, phase-change kinetics, etc.), (c) kinetic-theory modeling, (d) Monte-Carlo simulations of molecular flows, (e) the molecular kinetics of species, isotope, and particle separating gas flows, (f) energy-relaxation, phase-change, and ionization processes in gases, ( $g$ ) molecular beam techniques, and (h) low-density aerodynamics, to name the major ones.

This field, having always been strongly international in its makeup, had its beginnings in the early development of the kinetic theory of gases, the production of high vacuums, the generation of molecular beams, and studies of gas-surface interactions. A principal factor eventually solidifying the field was the need, beginning approximately twenty years ago, to develop a basis for predicting the aerodynamics of space vehicles passing through the upper reaches of planetary atmospheres. That factor has continued to be important, although to a decreasing extent; its importance may well increase again, now that the USA Space Shuttle vehicle is approaching operating status.

A second significant force behind work in this field is the strong commitment on the part of several nations to develop better means for enriching uranium for use as a fuel in power reactors. A third factor, and one which surely will be of long term importance, is that fundamental developments within this field have resulted in several significant spinoffs. A major example in this respect is the development of the nozzle-type molecular beam, where such beams represent a powerful means for probing the fundamentals of physical and chemical interactions between molecules.

Within these volumes is offered an important sampling of rarefied gas dynamics research currently under way. The papers included have been selected on the basis of peer and editor review, and considerable effort has been expended to assure clarity and correctness.

Published in 1981,1224 pp., 6×9, illus., $\$ 65.00 \mathrm{Mem} ., \$ 109.00$ List 